\title{
Coming together during viral assembly
}

\author{
Christof Hepp and Nicole C. Robb
}

This month's Under the Lens discusses how super-resolution microscopy has been used to answer fundamental questions about the assembly mechanisms of enveloped viruses. What, if anything, helps the major components of a virus particle find each other?

Enveloped viruses are surrounded by a lipid bilayer, which is formed as nascent virions bud through cellular membranes. Virus assembly at the plasma membrane is an essential part of the viral life cycle, and its regulation is a potential target for antiviral therapies. It is generally accepted that the accumulation of viral matrix proteins at the plasma membrane is sufficient to drive the formation of virus-like particles (VLPs), while viral glycoproteins are incorporated into the particles to mediate host cell entry. Viral glycoproteins are thought to be directed to assembly sites through interactions with viral matrix proteins; however, precise information on the distribution, organization and dynamics of the viral components is needed to determine whether this association is random or the result of specific regulatory interactions. Two recent studies have sought to address

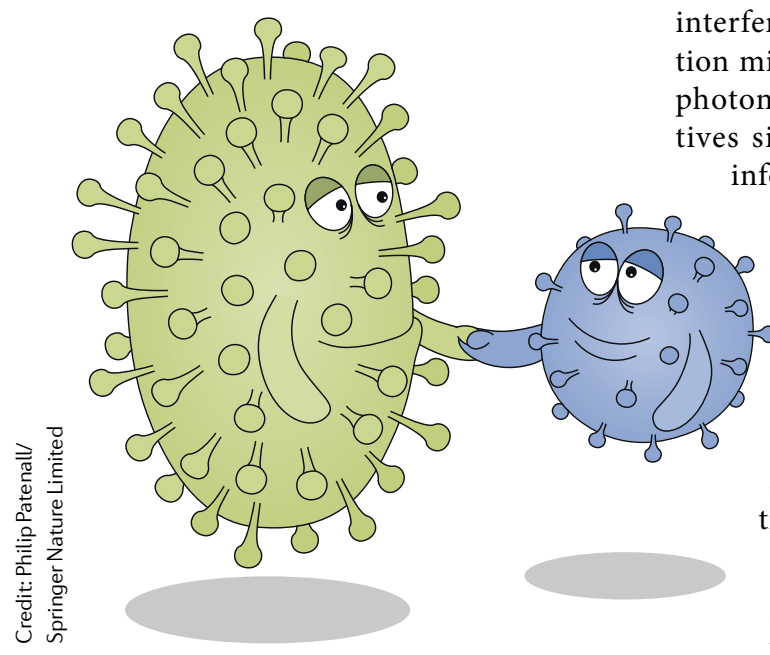

In a separate study, Buttler et al. ${ }^{2}$ used terferometric photoactivation localization microscopy (iPALM), which uses the ton signal collected from two objeces simultaneously to gain precise $3 \mathrm{D}$

information, to obtain super-resolved images of cell-associated HIV-1 particles. During HIV-1 infection, the matrix domain of the Gag polyprotein oligomerizes into a lattice to drive the formation of VLPs at assembly sites, and viral envelope (Env) glycoproteins assemble within this lattice ${ }^{3}$. Steric trapping of the long cytoplasmic tail domain of Env (Env-CT) between Gag proteins is thought to retain Env at assembly sites, and because only a small number (7-14 molecules) of Env glycoproteins are present on individual released particles, this incorporation is likely to be tightly regulated. The authors showed that Env predominantly clusters at the neck region of VLPs, suggesting that Env is incorporated into the Gag lattice at a late time point during assembly. Spinning disc confocal imaging of infected cells showed that Env was predominantly retained intracellularly, possibly to prolong incorporation into VLPs and limit its density on individual virus particles. Furthermore, single-particle tracking revealed that the Env-CT domain decreased Env mobility within the plasma membrane, supporting a model in which steric trapping supports the incorporation of Env into nascent particles. Whereas Liu et al's study provides evidence for stochastic incorporation of glycoproteins into $\mathrm{NiV}$ virions, Buttler et al.'s results point to a tightly regulated assembly pathway that limits cell surface exposure and the incorporation of Env into HIV-1 virions.

In summary, the comparison of these two studies highlights important differences in enveloped virus assembly mechanisms, and illustrates the strength of super-resolution microscopy techniques in taking advantage of precise spatial information to discover subtle regulation mechanisms that would not be observed using conventional light microscopy.

Christof Hepp* and Nicole C. Robb* Clarendon Laboratory, Department of Physics, University of Oxford, Oxford, UK. *e-mail: underthelens@bioch.ox.ac.uk https://doi.org/10.1038/s41579-018-0102-4

1. Liu, Q. et al. A stochastic assembly model for Nipah virus revealed by super-resolution microscopy. Nat. Commun. 9, 3050 (2018).

2. Buttler, C. A. et al. Single molecule fate of HIV-1 envelope reveals late-stage viral lattice incorporation. Nat. Commun. 9, 1861 (2018).

3. Freed, E. O. HIV-1 assembly, release and maturation. Nat. Rev. Microbiol. 13, 484 (2015).

\section{Competing interests}

The authors declare no competing interests. 